Percutaneous flexible endoscopic necrosectomy for a retroperitoneal abscess
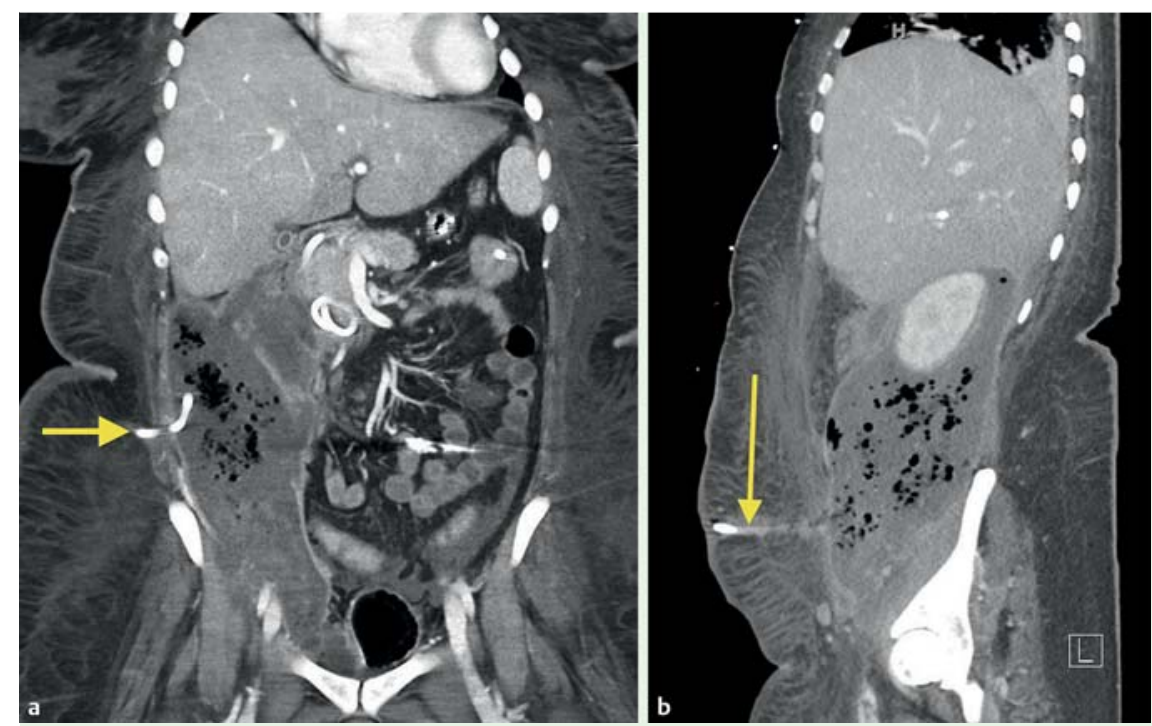

Fig. 1 a Coronal CT scan demonstrating the rostral percutaneous drain (arrow) within the abscess. b Sagittal CT scan demonstrating the caudal percutaneous drain (arrow) within the abscess.

Increasing evidence supports endoscopic transluminal retroperitoneal debridement for the management of walled-off

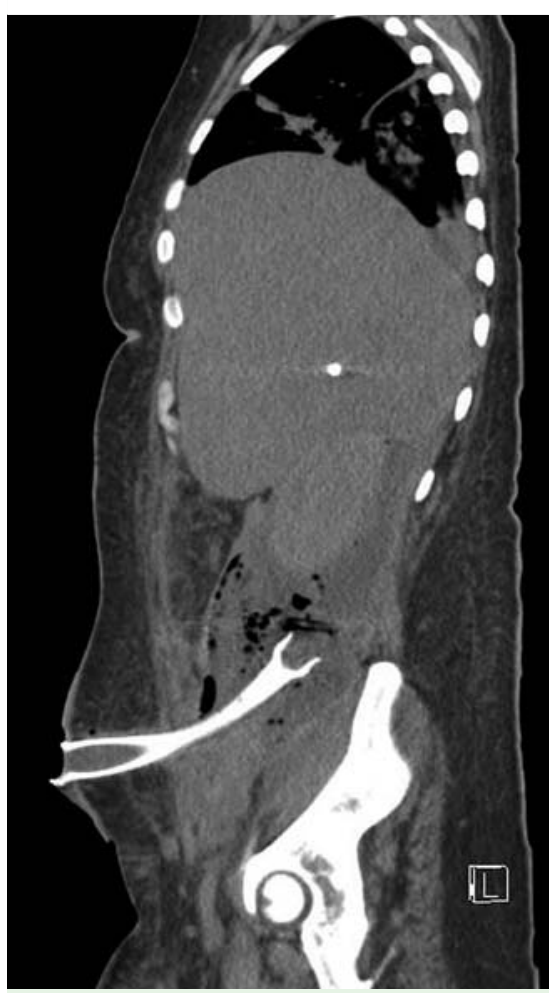

Fig. 2 Sagittal CT scan depicting the percutaneous deployment of the fully covered self-expandable metallic stent (FCSEMS) within the abscess. pancreatic necrosis $[1,2]$. However, this approach is not feasible for cavities that do not approximate the gastrointestinal lumen. Yamamoto et al. recently demonstrated a method of percutaneous endoscopic necrosectomy (PEN) requiring 11 procedures [3]. We demonstrate an alternative, more efficient method using PEN plus daily irrigation with hydrogen peroxide to efficiently manage a retroperitoneal abscess.

A 30-year-old woman was referred to our institution for management of a $15 \times 8 \mathrm{~cm}$ right retroperitoneal abscess extending from the subhepatic space into the pelvis which had failed to improve after insertion of two percutaneous drains ( $\bullet$ Fig. 1). Six weeks earlier she had suffered a type I duodenal perforation (lateral duodenal wall) during attempted endoscopic retrograde cholangiopancreatography (ERCP) performed for choledocholithiasis. Because biliary cannulation failed, the patient underwent percutaneous transhepatic biliary drainage.

The abscess was not visualized on upper endoscopic ultrasonography and hence the decision was made to proceed with PEN. Under fluoroscopic guidance, a guidewire was inserted through the caudal drain and exchanged for an esophageal $18 \times 103 \mathrm{~mm}$ fully covered self-expandable metallic stent ( $\bullet$ Fig. 2). The stent was placed into the abscess and secured at three sites with two 1-0 nonab- 


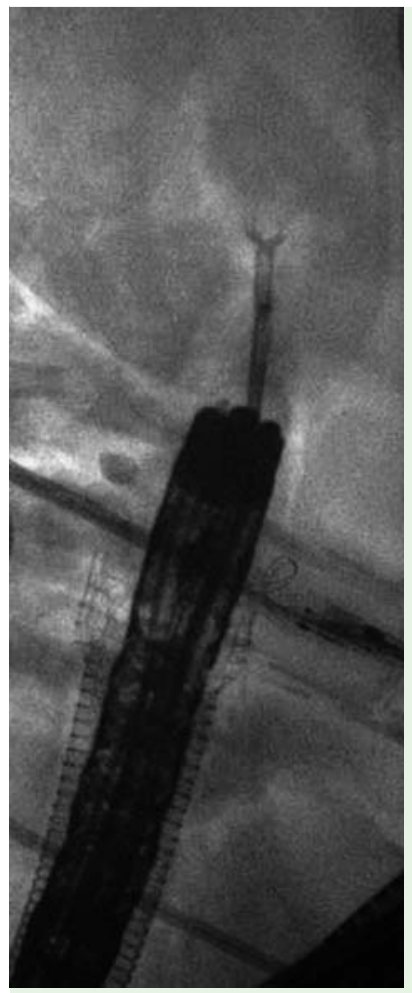

Fig.4 Fluoroscopic image of the gastroscope passing through the stent. The forceps are being used to remove necrotic debris.

sorbable sutures and covered with a stoma bag ( Fig.3). The rostral drain was noted to be occluded and was exchanged for a 16-Fr balloon-type gastrostomy tube.

The following day, under minimal sedation, PEN was performed with an adult gastroscope passed through the stent using $\mathrm{CO}_{2}$ insufflation. Copious amounts of solid and purulent necrotic debris were extracted using retrieval forceps, snares, baskets, and Roth nets ( Fig.4, - Video 1). Between procedures, $300 \mathrm{~mL}$ hydrogen peroxide $(0.3 \%)$ was injected through the gastrostomy tube twice daily to chemically debride and lavage the necrosis. Only four procedures were required over 8 days to entirely clear the necrotic debris ( Fig.5). The stent and gastrostromy tube were removed and the patient was discharged home to return in

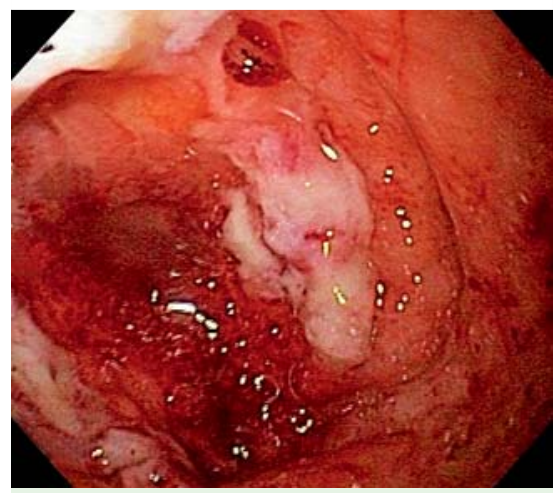

Fig. 5 Endoscopic view of the cavity, which became lined with healthy granulation tissue after only 8 days.

4 weeks for successful ERCP and stone extraction.

We demonstrate a novel method of managing a retroperitoneal abscess not amenable to transluminal drainage. PEN allows unwell patients to undergo multiple procedures using only minimal sedation. Additionally, twice daily irrigation with hydrogen peroxide as a chemically debriding agent appears to be a safe and effective method to expedite resolution.

\section{Video 1}

Insertion and positioning of the fully covered selfexpandable metallic stent, positioning of gastrostomy tube, and subsequent necrosectomy.

\section{Endoscopy_UCTN_Code_TTT_1AS_2AC}

Competing interests: Payal Saxena has received consulting fees from Boston Scientific and has received research support from Cook Medical.

Mouen A. Khashab is a consultant for Boston Scientific and Olympus America and has received research support from Cook Medical.

All other authors have no relevant disclosures.
Vivek Kumbhari, Andrew C. Storm, Alan H. Tieu, Payal Saxena, Ahmed A. Messallam, Mohamad H. El Zein, Alba Azola, Mouen A. Khashab, Patrick I. Okolo III

Division of Gastroenterology and Hepatology, Department of Medicine, The Johns Hopkins Medical Institutions, Baltimore, Maryland, USA

\section{References}

1 Yasuda I, Nakashima M, Iwai T et al. Japanese multicenter experience of endoscopic necrosectomy for infected walled-off pancreatic necrosis: the JENIPaN study. Endoscopy 2013; 45: 627-634

2 Abdelhafez M, Elnegouly M, Hasab Allah MS et al. Transluminal retroperitoneal endoscopic necrosectomy with the use of hydrogen peroxide and without external irrigation: a novel approach for the treatment of walled-off pancreatic necrosis. Surg Endosc 2013; 27: 3911 - 3920

3 Yamamoto $N$, Isayama $H$, Takahara $N$ et al. Percutaneous direct-endoscopic necrosectomy for walled-off pancreatic necrosis. Endoscopy 2013; 45 (Suppl. 02): E44-45

Bibliography

DOI http://dx.doi.org/

10.1055/s-0034-1377360

Endoscopy 2014; 46: E340-E341

(c) Georg Thieme Verlag KG

Stuttgart · New York

ISSN 0013-726X

Corresponding author

Patrick I. Okolo III, MD

Johns Hopkins Hospital

1800 Orleans St, Suite 7125 G

Baltimore, MD 21205

USA

pokolo2@jhmi.edu 